\title{
On Stability Switches and Bifurcation of the Modified Autonomous Van der Pol-Duffing Equations via Delayed State Feedback Control
}

\author{
Tiao-Yang Cai ${ }^{1}$, Hui-Long Jin ${ }^{1, *}$, Hong Yu ${ }^{1}$ and Xiang-Peng Xie ${ }^{2}$ \\ 1 Collage of Engineering, Hebei Normal University, Shijiazhuang 050024, China; \\ chaoyang712@hebtu.edu.cn (T.-Y.C.); yuhong6411@hebtu.edu.cn (H.Y.) \\ 2 Institute of Advanced Technology, Nanjing University of Posts and Telecommunications, \\ Nanjing 210003, China; xiexp@njupt.edu.cn \\ * Correspondence: JHL981@hebtu.edu.cn
}

Citation: Cai, T.-Y.; Jin, H.-L.; Yu, H.; Xie, X.-P. On Stability Switches and Bifurcation of the Modified

Autonomous Van der Pol-Duffing Equations via Delayed State Feedback Control. Symmetry 2021, 13, 2336. https://doi.org/10.3390/sym13122336

Academic Editors: Jing Li and Zhaohui Zhang

Received: 11 October 2021

Accepted: 1 December 2021

Published: 6 December 2021

Publisher's Note: MDPI stays neutral with regard to jurisdictional claims in published maps and institutional affiliations.

Copyright: (c) 2021 by the authors. Licensee MDPI, Basel, Switzerland. This article is an open access article distributed under the terms and conditions of the Creative Commons Attribution (CC BY) license (https:/ / creativecommons.org/licenses/by/ $4.0 /)$.

\begin{abstract}
This paper considers the Modified Autonomous Van der Pol-Duffing equation subjected to dynamic state feedback, which can well characterize the dynamic behaviors of the nonlinear dynamical systems. Both the issues of local stability switches and the Hopf bifurcation versus time delay are investigated. Associating with the $\tau$ decomposition strategy and the center manifold theory, the delay stable intervals and the direction and stability of the Hopf bifurcation are all determined. Specifically, the computation of purely imaginary roots (symmetry to the real axis), the positive real root formula for cubic equation and the sophisticated bilinear form of adjoint operators are proposed, which make the calculations mentioned in our discussion unified and simple. Finally, the typical numerical examples are shown to illustrate the correctness and effectiveness of the practical technique.
\end{abstract}

Keywords: stability switches; Hopf bifurcation; $\tau$-decomposition method; center manifold theory

\section{Introduction}

Since the effective characterization of the nonlinear phenomenons, nonlinear electronic circuits have been extensively studied in the last decades by scientists, engineers and physicists [1]. The early substantial achievement can be seen in the Chua's circuit in the mid-1960s. Since then, different nonlinear circuits were introduced by many scholars. For example, Shinriki et al. introduced a circuit representing the modified Van der Pol oscillator (MVPO) in [2]. King and Gaito derived a nonlinear circuit from the (MVPO) in [3]. Furthermore, Matouk et al. gave simple modification to the Van der Pol-Duffing circuit in [4]. It is noted that this circuit represents the autonomous Van der Pol-Duffing (AVPD) oscillator and is equivalent to Chua's autonomous circuit but with a cubic nonlinear element.

Generally speaking, the Modified Autonomous Van der Pol-Duffing (MAVDP) equations is described by the following form:

$$
\begin{aligned}
& \dot{x}(t)=-v\left[x^{3}(t)-\mu x(t)-y(t)\right] \\
& \dot{y}(t)=x(t)-\alpha y(t)-z(t) \\
& \dot{z}(t)=\beta y(t)
\end{aligned}
$$

where $\alpha, \beta, v$ are all positive reals.

Indeed, the local codimension bifurcations are investigated in a Van der Pol-Duffing circuit with parallel resistor in [5]. In addition, a complete description of the regions with multiple small periodic solutions arise through the Hopf bifurcations has been provided. In [6], Fan investigated the existence of chaos in a modified Van der Pol-Duffing system by a rigorous verification by using topological horseshoe theory. Recently, the dynamics of a driven Van der Pol-Duffing oscillator circuit was investigated in [7]. Both the existence 
of higher-dimensional chaotic orbits and transient dynamics are addressed. Moreover, Han et al. propose two novel bursting patterns in modified Van der Pol-Duffing circuit system in [8]. These two bursting patterns are classified via "supHopf/supHopf" hysteresis loop. Meanwhile, by the multiple timescales method, the pure and impure quadratic damping of a hybrid Rayleigh-Van der Pol-Duffing oscillator are investigated in [9], and the various resonance states are considered exhaustively. A model of human walking is represented by a modification of hybrid Van der Pol-Duffing-Rayleigh oscillator in [10]. A stability analysis of the proposed oscillator has been performed by using the energy balance method and the Lindstedt-Poincare perturbation technique. A unified Van der Pol-Duffing circuit is used to design a memristor-based chaotic circuit by introducing an ideal and active flux-controlled memristor in [11]. Rajagopa et al. deal with a new modified hyperchaotic Van der Pol-Duffing (MVPD) snap oscillator in [12]. With the help of Lyapunov exponents, various dynamical properties are investigated. Both the stability analysis and the existence of the Hopf bifurcation is established. More recently, the generation mechanism of vibration in a driven Van der Pol-Duffing oscillator is explored in [13], and it is proved that the pitchfork bifurcation delay phenomenon deserves a prominent role in the occurrence of the mixed mode vibrations. A fractional order eco-epidemic model is addressed in [14], and the local stability analysis conditions for the existence of Hopf bifurcation are established.

For obtaining the desired dynamical behaviors, the original system in (1) can be subject to state feedback control in [15]. Moreover, the existing control methods can be classified, mainly, into two categories: the static state feedback control mode is the one in which the value of the input $u$ at time $t$ is a function of the value, at this time, of the state $x(t)$. In particular, one is interested in control laws of the form

$$
u(t)=K x(t)
$$

Secondly, the dynamic state-feedback control mode is the one in which the value of the input $u$ at time $t$ is a function of the value, at this time, of the state $x(t)$ of a new input $x_{t}(\theta)$ and of a new set of state variables. In particular, the delayed state can be chosen as a candidate, and the control laws can be described by equations of the form

$$
u(t)=K[x(t-\tau)-x(t)], \tau \geq 0
$$

To the best of the authors' knowledge, the research on the mathematical model of MAVDP circuits with delayed feedback regulation are novelty ones, since the specific analysis of the intrinsic quality is benefit for the design of the physical circuit. It is especially suitable for the circuits with the time delay units, which are designed by the filters of $\mathrm{T}$ type LCL, as is pointed out in [16]. Meanwhile, the delayed circuits also can be viewed as nonlinear systems. It is worthy to analyze the dynamic nonlinear systems that are applicable for real world problems. In this paper, we will give a rigorous verification for stability switches and the Hopf bifurcation in the modified Van der Pol-Duffing system with delayed feedback. In sum, the novelties of this paper lie in the following aspects:

- $\quad$ Both the delay independent stable condition and the delay dependent stable interval of MAVPD in (2) are included in the framework of the $\tau$ decomposition method.

- Based on the adjoint operator theory, a lite bilinear form is introduced to reduce the amount of calculations on projection and adjoint eigenvectors.

- By solving the initial value problem of ordinary differential equation, a sophisticated procedure is addressed for the computation of Hopf bifurcation.

\section{Problem Statement and Preliminaries}

In this section, we state the MAVPD in a delay differential equation form and introduce the framework to deal with the issues of local analysis and the nonlinear dynamics. It includes the determination of the stability switches and the bifurcating periodic solutions associated with the $\tau$ decomposition technique and the center manifold theory. 
Subjected to the dynamic state feedback control law, the original MAVPD equation in (1) can be written as

$$
\begin{aligned}
& \dot{x}(t)=-v\left[x^{3}-\mu x-y\right]+K[x(t-\tau)-x(t)] \\
& \dot{y}(t)=x-\alpha y-z \\
& \dot{z}(t)=-\beta y
\end{aligned}
$$

It is a conventional way to get the equilibrium points of system (2), and they are

$$
\left\{\begin{array}{l}
E_{0}=(0,0,0) \\
E_{+}=(\sqrt{\mu}, 0, \sqrt{\mu}) \\
E_{-}=(-\sqrt{\mu}, 0,-\sqrt{\mu})
\end{array}, \mu>0 \text { and } E_{0}=(0,0,0), \mu<0 .\right.
$$

With the notation of $X(t)=[x(t), y(t), z(t)]^{T}$, the system (2) can be stated in the matrix form around the equilibrium point of $E_{0}$

$$
\dot{X}=A X(t)+B X(t-\tau)+F(X)
$$

with the corresponding Jacobian matrices such that

$$
A=\left[\begin{array}{ccc}
\mu v-K-3 v x_{0}^{2} & v & 0 \\
1 & -\alpha & -1 \\
0 & \beta & 0
\end{array}\right] \text { and } B=\left[\begin{array}{lll}
K & 0 & 0 \\
0 & 0 & 0 \\
0 & 0 & 0
\end{array}\right] \text { and } F(X)=\left[\begin{array}{c}
-v x^{3}(t) \\
0 \\
0
\end{array}\right]
$$

There is no doubt that the local stability of the system is dependent on the linear part of (4), and the associating characteristic equation is

$$
C E(\lambda)=\lambda^{3}+a \lambda^{2}+b \lambda+c-K\left(\lambda^{2}+\alpha \lambda+\beta\right) e^{-\tau \lambda}
$$

with

$$
\begin{aligned}
& a=\alpha+K+3 v x_{0}^{2}-\mu v \\
& b=\beta+\alpha K+3 \alpha v x_{0}^{2}-\alpha \mu v-v, \\
& c=\beta K+3 \beta v x_{0}^{2}-\beta \mu v .
\end{aligned}
$$

\section{The $\tau$ Decomposition Method}

The $\tau$ decomposition method is a better choice for the local asymptotic stability analysis of systems with time delay. This technique treats the time delay as the variable parameter. In [17], this technique involves firstly dividing the delay $\tau$ axis into intervals, and the same stability character prevails within each interval. Then the crossing behaviors over the interval endpoints can determine the stability switches. Thus, there exist mainly two aspects of work. One is to calculate the interval endpoints on the delay axis, and the other is to determine whether the crossing of the endpoint is stable or not. The former one can resort to the computation of purely imaginary roots (PIR) of the system's characteristic equation. The conjugate complex roots appear in pairs, i.e., they are symmetrical to the real axis in the complex plane. Thus, we can only consider the positive PIRs, and the later one is related to the cross directions around the PIRs. Fortunately, the number of PIRs of the transcendental equation is finite, and the cross direction on every PIR is independent of $\tau$. Now the corresponding results are introduced.

Lemma 1. For the transcendental equation in (6), the purely imaginary roots (PIRs) are the positive real roots (PRRs) of auxiliary polynomial in $\omega^{2}$

$$
W\left(\omega^{2}\right)=\omega^{6}+p \omega^{4}+q \omega^{2}+r
$$


with

$$
\begin{aligned}
& p=a^{2}-2 b-K^{2} \\
& q=b^{2}-2 a c+2 K^{2} \beta-K^{2} \alpha^{2} \\
& r=c^{2}-K^{2} \beta^{2}
\end{aligned}
$$

Before we proceed, some simple trivial cases should be excluded by the following assumption.

Assumption 1. The characteristic Equation (6) corresponds to no zero and multiple PIRs.

Further, the auxiliary polynomial in (8) tells us that the PIRs of (2) in (6) are quite related to the positive real roots (PRR) of cubic polynomial in (8). Thus, a targeted result on the positive real roots is deduced.

Lemma 2. For the cubic equation

$$
\lambda^{3}+p \lambda^{2}+q \lambda+r=0,
$$

the positive real roots (PRR) are defined such that:

(i) There is one distinct $P R R$, if and only if

$$
r<0,\left\{\begin{array}{l}
\Delta>0 \\
\Delta<0, \Delta_{1}<0 \\
\Delta=0, p q>0
\end{array}\right\}
$$

and it is

$$
\lambda_{1}=S+T-\frac{p}{3} .
$$

(ii) There are two distinct PRRs, if and only if

$$
\Delta<0, \Delta_{2}>0, r>0
$$

and they are

$$
\begin{aligned}
& \lambda_{2}=w S+w^{2} T-\frac{p}{3} \\
& \lambda_{3}=w^{2} S+w T-\frac{p}{3} .
\end{aligned}
$$

(iii) There exist three distinct PRRs, if and only if

$$
\Delta<0, \Delta_{1}>0, r<0
$$

and they are (12) and (14), in which

$$
\begin{array}{ll}
w=\frac{-1+\sqrt{3} j}{2}, & S=\sqrt[3]{-\frac{Q}{2}+\sqrt{\Delta}} \\
P=q-\frac{p^{2}}{3}, & T=\sqrt[3]{-\frac{Q}{2}-\sqrt{\Delta}} \\
Q=r+\frac{2 p^{3}}{27}-\frac{p q}{3}, & \Delta_{1}=-\frac{p}{3}-\sqrt{p^{2}-3 q} \\
\Delta=\frac{Q^{2}}{4}+\frac{p^{3}}{27}, & \Delta_{2}=-\frac{p}{3}+\sqrt{p^{2}-3 q} .
\end{array}
$$

Proof. See in the Appendix A.

Meanwhile, the issue of cross directions over PIRs is relatively concise according to the discussion in [18], and it is summarized in the next statement.

For convenience, we call those $\omega_{i}$ s such that

$$
\omega_{1}>\omega_{2}>\omega_{3}
$$

are the cross frequencies (CF). Moreover, the cross directions (CD) over those $\mathrm{CFs}$ are summarized below. 
Remark 1. As is clearly shown in [18], the CD at the largest CF of $\omega_{1}$ is always to the right (the unstable crossing), at $\omega_{2}$ is always to the left (the stable crossing), and at $\omega_{3}$ is still unstable crossing.

On the other hand, the eigen-decomposition theory tells us that, the divide of the subspace corresponding to PIR $j \omega_{0}$ needs the eigen-vector and the adjoint vector associating with $\pm j \omega$. Therefore, we first introduce a distinguished bilinear form for the eigenvalue decomposition in the analysis of nonlinear dynamics of (2).

Lemma 3. For $X$ and $Y$ are the solutions of the linearized system in (2) and its adjoint system

$$
\dot{Y}(s)=A^{T} Y(s)+B^{T} Y(s+\tau),
$$

respectively. The value of bilinear form

$$
I=\bar{Y}^{T}(0) X(0)+\int_{-\tau}^{0} \bar{Y}^{T}(\xi+\tau) B X(\xi) d \xi
$$

can be called the inner product $\langle Y, X\rangle$.

\section{Main Results}

\subsection{The Issue of Linear Analysis}

In this section, the phenomenon of stability switch is investigated in a unified manner, and the occurrence of Hopf bifurcation is revealed as well.

Before we proceed, necessary statements and definitions should be introduced according to the discussion mentioned above.

As is indicated in Lemma 2, we can obtain all the PIRs analytically depending on the three number conditions. In practice, every $j \omega_{i}$ corresponds to a series of critical delays,

$$
T_{i}=\left\{\tau_{i k} \mid \tau_{i k}=\tau_{0 i}+\frac{2 k \pi}{\omega_{i}}\right\}, \quad \begin{aligned}
& i=1,2,3 . \\
& k=0,1,2,3, \ldots .
\end{aligned}
$$

with $\tau_{0 i}=\frac{\theta_{i}}{\omega_{i}}$ being the smallest positive real such that

$$
\theta_{i}=\left\{\begin{array}{c}
\angle\left[\frac{\beta-\omega^{2}+\alpha \omega j}{c-a \omega^{2}+\left(b \omega-\omega^{3}\right) j}+\pi\right], 0<\angle \frac{\beta-\omega^{2}+\alpha \omega j}{c-a \omega^{2}+\left(b \omega-\omega^{3}\right) j}<\pi \\
\angle\left[\frac{\beta-\omega^{2}+\alpha \omega j}{c-a \omega^{2}+\left(b \omega-\omega^{3}\right) j}-\pi\right], \pi<\angle \frac{\beta-\omega^{2}+\alpha \omega j}{c-a \omega^{2}+\left(b \omega-\omega^{3}\right) j}<2 \pi
\end{array}\right.
$$

Definition 1. The MAVPD system is the initial stable means that the roots of the characteristic equation of the system in (6) are all with negative real parts when $\tau=0$.

Theorem 1. For the initial stable MAVPD system in (2), the local dynamics satisfy:

(i) The delay dependent stable interval for $\tau \in\left[0, \tau_{10}\right)$, with the one PIR condition in (11).

(ii) The delay $N+1$ dependent stable interval for $N$ such that

$$
\begin{gathered}
\max \left\{N \mid \frac{\tau_{2 N}-\tau_{20}}{\tau_{1 N}-\tau_{10}}<\frac{\omega_{1}}{\omega_{2}}\right\}, \text { and } \\
\max \left\{N \mid\left(\tau_{2 N}-\tau_{20}\right) \omega_{2}<\left(\tau_{1 m}-\tau_{10}\right) \omega_{1}+\left(\tau_{3 n}-\tau_{30}\right) \omega_{3}\right\}
\end{gathered}
$$

with the two and three PIRs conditions in (13) and (15), respectively, where $N=0,1,2, \cdots$ and $m+$ $n \leq N$.

Moreover, there exists a Hopf bifurcation of system (2) when the delay passes through those critical delays $\tau_{i j}$ s from stable (unstable) interval into unstable (stable) interval.

Proof. Associating with the initial stable MAVPD and the one PIR condition which corresponds to unstable crossing, the system becomes unstable after $\tau_{10}$, and it will never return, for all the crossings associated with $\omega_{1}$ are unstable. Then, the result for only one 
delay interval is obtained. In the case of three-PIR condition, the delay stable intervals must satisfy

$$
\frac{\left(\tau_{2 N}-\tau_{20}\right) \omega_{2}}{2 \pi}<\frac{\left(\tau_{1 m}-\tau_{10}\right) \omega_{1}}{2 \pi}+\frac{\left(\tau_{3(N-m)}-\tau_{30}\right) \omega_{3}}{2 \pi}
$$

in which

$$
\frac{\left(\tau_{2 N}-\tau_{20}\right) \omega_{2}}{2 \pi}
$$

are the times of stable crossings and

$$
\frac{\left(\tau_{1 m}-\tau_{10}\right) \omega_{1}}{2 \pi}+\frac{\left[\tau_{3(N-m)}-\tau_{30}\right] \omega_{3}}{2 \pi}
$$

are the times of unstable crossings. The condition of (21) guarantees the stable crossing comes before the unstable crossing. The two-PIR condition is a special case mentioned above. Therefore, we get the stability switches.

Corollary 1. For the intimal unstable MVPD system in (2), it is delay N interval dependent stable for $N$ such that

$$
\begin{gathered}
\max \left\{N \mid \frac{\tau_{2 N}-\tau_{20}}{\tau_{1 N}-\tau_{10}}<\frac{\omega_{1}}{\omega_{2}}\right\}, \text { and } \\
\max \left\{N \mid\left(\tau_{2 N}-\tau_{20}\right) \omega_{2}<\left(\tau_{1 m}-\tau_{10}\right) \omega_{1}+\left(\tau_{3 n}-\tau_{30}\right) \omega_{3}\right\}
\end{gathered}
$$

with the two- and three-PIR conditions in (13) and (15), respectively, where $N=0,1,2, \cdots$ and $m+$ $n \leq N$.

Moreover, there exists a Hopf bifurcation of system (2) when the delay passes through those critical delays $\tau_{i j}$ s from stable (unstable) interval into unstable (stable) interval.

Remark 2. With the discussion mentioned above, the Hopf bifurcation occurs when the delay passes through those critical delays $T_{i k}$ from stable (unstable) interval into unstable (stable) interval.

\subsection{The Issue of Periodical Solutions}

In this section, we will rescale the MAVPD equation in (2) by $t_{\text {new }}=\frac{t_{\text {old }}}{\tau}$. Then, the time delay is tacked with the bifurcating parameter, and we have

$$
\dot{X}(t)=\tau A X(t)+\tau B X(t-1)+F\left(X_{t}\right)
$$

with

$$
F=\left[\begin{array}{c}
-\tau v X_{t}^{(1)}(0)^{3} \\
0 \\
0
\end{array}\right]
$$

The abstract differential equation is

$$
\dot{X}_{t}=L X_{t}+R X_{t}
$$

The linear operator $L$ satisfies

$$
L \varphi(\theta)=\left\{\begin{array}{l}
\frac{d \varphi}{d \theta}, \quad-1 \leq \theta<0 \\
\int_{-1}^{0} d \eta(s) \varphi(s), \theta=0
\end{array}\right.
$$

with

$$
d \eta(\theta)=\tau[A \delta(\theta)+B \delta(\theta+1)],
$$

and $\delta(\cdot)$ is the Dirac delta function. 
Then, the nonlinear part is as follows:

$$
R_{t}(\theta)=\left\{\begin{array}{l}
{\left[\begin{array}{l}
0 \\
0 \\
0
\end{array}\right], \quad-1 \leq \theta<0} \\
{\left[\begin{array}{c}
f_{1} \\
0 \\
0
\end{array}\right], \quad \theta=0}
\end{array}\right.
$$

with $f_{1}=-v X_{t}^{(1)}(0)^{3}$.

Moreover, the linear operator $L$ is adjoint with the operator of $L^{*}$ such that

$$
L^{*} \psi(\theta)=\left\{\begin{array}{l}
-\frac{d \psi}{d \theta}, \quad 0<\theta \leq 1 \\
\int_{-1}^{0} d \eta^{T}(t) \psi(-t), \theta=0
\end{array}\right.
$$

associating with the simplified inner product by Lemma 3,

$$
<\psi, \varphi>=\bar{\psi}^{T}(0)\left[I+e^{-j \omega_{0}} B\right] \varphi(0) .
$$

Therefore, we can determine the adjoint vector for constructing the center manifold through the following statement.

Lemma 4. The eigenvectors of linear operator $L$ and its adjoint $L^{*}$ associate with $j \omega_{0}$ such that

$$
q(\theta)=\left[\begin{array}{c}
1 \\
q_{2} \\
q_{3}
\end{array}\right] e^{j \omega_{0} \theta}
$$

and

$$
q^{*}(s)=D\left[\begin{array}{c}
1 \\
q_{2}^{*} \\
q_{3}^{*}
\end{array}\right] e^{j \omega_{0} s}
$$

satisfying $<q, q^{*}>=1,<\bar{q}, q^{*}>=0$, in which

$$
\begin{aligned}
& q_{2}=j \omega_{0} \chi \\
& q_{3}=\tau \beta \chi \\
& q_{2}^{*}=-j \omega_{0} v \bar{\chi} \\
& q_{3}^{*}=-\tau v \bar{\chi} \\
& D=\left[1+\bar{q}_{2} q_{2}^{*}+\bar{q}_{3} q_{3}^{*}+\tau K e^{j \omega_{0}}\right]^{-1}
\end{aligned}
$$

with

$$
\chi=\frac{\tau}{\tau^{2} \beta-\omega_{0}^{2}+\tau \alpha \omega_{0} j}
$$

Proof. As the eigenvector of differential operator $L$ associating with $j \omega_{0}$ such that $L q(\theta)=$ $j \omega_{0} q(\theta)$, we have

$$
q(\theta)=q(0) e^{j \omega \theta}
$$

Moreover, $L q(0)=\int_{-1}^{0} d \eta(s) q(s)=j \omega_{0} q(0)$ gives the matrix equation

$$
\tau A q(0)+\tau B q(0) e^{-j \omega_{0}}=j \omega_{0} q(0) .
$$


Thus,

$$
q(\theta)=\left[\begin{array}{c}
1 \\
\frac{j \omega_{0} \tau}{\tau^{2} \beta-\omega_{0}^{2}+\tau \alpha \omega_{0} j} \\
\frac{\tau^{2} \beta}{\tau^{2} \beta-\omega_{0}^{2}+\tau \alpha \omega_{0} j}
\end{array}\right] e^{j \omega_{0} \theta} .
$$

On the other hand, the adjoint matrix equation from (26) is

$$
\tau A^{T} q_{0}^{*}(0)+\tau B^{T} q_{0}^{*}(0) e^{j \omega_{0}}=-j \omega_{0} q_{0}^{*}(0) .
$$

We obtain an adjoint eigenvector

$$
q_{0}^{*}(s)=\left[\begin{array}{c}
1 \\
\frac{-\tau \nu \omega_{0} j}{\omega_{0}^{2}-\tau^{2} \beta+\tau \alpha \omega_{0} j} \\
\frac{-\tau^{2} v}{\omega_{0}^{2}-\tau^{2} \beta+\tau \alpha \omega_{0} j}
\end{array}\right] e^{j \omega_{0} s} .
$$

Additionally, the normalized eigenvector $q^{*}=D q_{0}^{*}$ should be such that $\left\langle q, D q_{0}^{*}\right\rangle=1$, and we have

$$
<q, q_{0}^{*}>=\left[\bar{q}_{0}^{* T}(0) q(0)+e^{j \omega_{0}} \bar{q}_{0}^{* T}(0) B q(0)\right]=\frac{1}{\bar{D}}
$$

Thus,

$$
D=\frac{1}{\overline{\left\langle q, q_{0}^{*}>\right.}}=\frac{1}{\left(1+\overline{q_{2}} q_{2}^{*}+\overline{q_{3}} q_{3}^{*}+\tau K e^{j \omega_{0}}\right)},
$$

and the orthogonality condition $\left\langle\bar{q}, q^{*}>=0\right.$ is easy to verify.

Applying the eigen-decomposition technique, we adopt the notations in [19] to divide the solution space of (23) into the center manifolds and stable ones.

$$
\left\{\begin{array}{l}
z(t)=<q^{*}, X_{t}> \\
w(t)=X_{t}-z q-\bar{z} \bar{q}
\end{array}\right.
$$

On the other, the center manifold theorem tells us that there exists a local manifold $w(t)$ such that

$$
w(t, \theta)=\frac{1}{2} w_{20}(\theta) z^{2}+w_{11}(\theta) z \bar{z}+\frac{1}{2} w_{02}(\theta) \bar{z}^{2}+\cdots
$$

The evolution of $z$ on $t$ gives

$$
\begin{aligned}
\dot{z}(t) & =\left\langle q^{*}, \dot{X}_{t}>=<q^{*}, L X_{t}>+<q^{*}, R X_{t}>=j \omega_{0} z(t)+\bar{q}^{* T}(0) R X_{t}(0)\right. \\
& =j \omega_{0} z+\bar{D}\left[1 \overline{q_{2}^{*}} \overline{q_{3}^{*}}\right]\left[\begin{array}{c}
-\tau v X_{t}^{(1)}(0)^{3} \\
0 \\
0
\end{array}\right]
\end{aligned}
$$

With the notation in (31), we have

$$
X_{t}^{(1)}(0)^{3}=\left[z q^{(1)}(0)+\bar{z} \bar{q}^{(1)}(0)+\frac{1}{2} w_{20}^{(1)}(0) z^{2}+w_{11}^{(1)}(0) z \bar{z}+\cdots\right]^{3}
$$

Therefore, we obtain

$$
\dot{z}=j \omega_{0} z+\frac{1}{2} g_{20} z^{2}+g_{11} z \bar{z}+\frac{1}{2} g_{02} \bar{z}^{2}+\frac{1}{2} g_{21} z^{2} \bar{z}+\cdots
$$

with

$$
g_{20}=g_{11}=g_{02}=0, g_{21}=-6 \tau \nu \bar{D}
$$


Remark 3. For applying the Hassard formula in [19],

$$
\left\{\begin{array}{l}
\mu_{2}=-\frac{1}{2} \frac{\operatorname{Re}\left[g_{21}\right]}{\alpha^{\prime}\left(\tau_{0}\right)} \\
\tau_{2}=-\frac{\operatorname{Im}\left[g_{21}\right]+2 \mu_{2} \omega^{\prime}(0)}{2 \omega_{0}} \\
\beta_{2}=\operatorname{Re}\left[g_{21}\right]
\end{array}\right.
$$

with $C_{1}(0)=\frac{j}{2 \omega_{0}}\left[g_{20} g_{11}-2\left|g_{11}\right|^{2}-\frac{1}{3}\left|g_{02}\right|^{2}\right]+\frac{g_{21}}{2}$. Accordingly, we have $C_{1}(0)=-3 \tau v \bar{D}$ in our discussion.

Theorem 2. In Formula (34), $\mu_{2}$ determines the direction of the Hopf bifurcation: if $\mu_{2}>0(<0)$, then the Hopf bifurcation is supercritical (subcritical); $\beta_{2}$ determines the stability of the bifurcating periodic solutions: the solutions are orbitally stable (unstable) if $\beta_{2}<0(>0)$; and $\tau_{2}$ determines the period of the bifurcating periodic solutions: the period increases (decreases) if $\tau_{2}>0(<0)$.

Based on all the discussion above, we can summarize the following unified Algorithm 1.

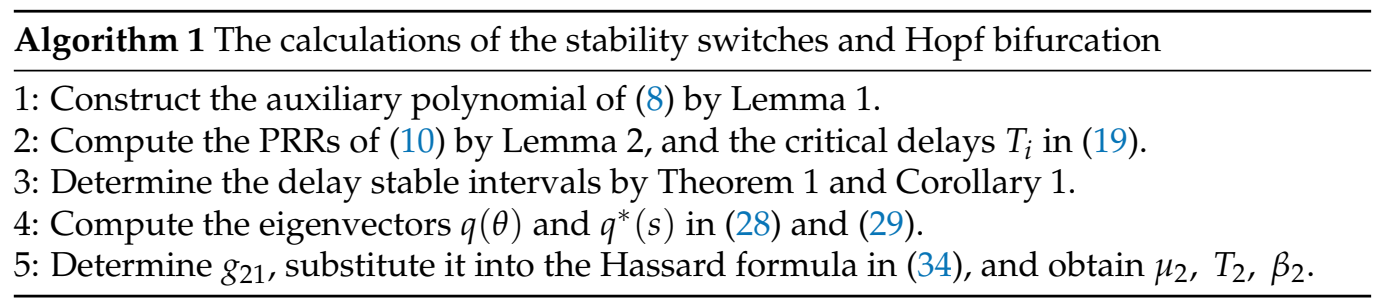

\section{Numerical Examples}

Example 1. The first example comes from [4] with $\alpha=3, \beta=200, \mu=0.1, v=100$. Thus, the $M A V P D$ with delayed feedback is

$$
\begin{aligned}
& \dot{x}(t)=-100\left[x^{3}-0.1 x-y\right]+10[x(t-\tau)-x(t)] \\
& \dot{y}(t)=x-1.6 y-z \\
& \dot{z}(t)=-200 y
\end{aligned}
$$
they are

It is easy to verify that there exist three equilibriums, which are mareked by blue symbols, and

$$
(0,0,0),( \pm 0.3162,0, \pm 0.3162)
$$

Firstly, we consider the origin point. The characteristic equation is

$$
C E(\lambda)=\lambda^{3}+3 \lambda^{2}+100 \lambda-\left(10 \lambda^{2}+30 \lambda+2000\right) e^{-\tau \lambda}
$$

By Lemma 1, we can see that it has only one PIR with $\omega_{1} j=j 11.9662$, and the critical delays are

$$
T_{1}=\left\{\tau_{1 k} \mid \tau_{1 k}=0.2363+\frac{2 k \pi}{11.9662}\right\}
$$

Unfortunately, all the critical delays correspond to the addition of unstable roots, which is shown in Figure 1. The curve is the rightmost root loci of the system in Example 1 around $E_{0}$. Since there exists a pair of symmetry conjugate roots with a positive real part for $\tau=0$, it adds two more symmetrical unstable roots for every critical delay. Thus, we conclude that the $E_{0}$ is an unstable equilibrium point for any $\tau \geq 0$.

Meanwhile, the characteristic equation around $E_{+}$is

$$
C E(\lambda)=\lambda^{3}+33 \lambda^{2}+190 \lambda+6000-\left(10 \lambda^{2}+30 \lambda+2000\right) e^{-\tau \lambda}
$$




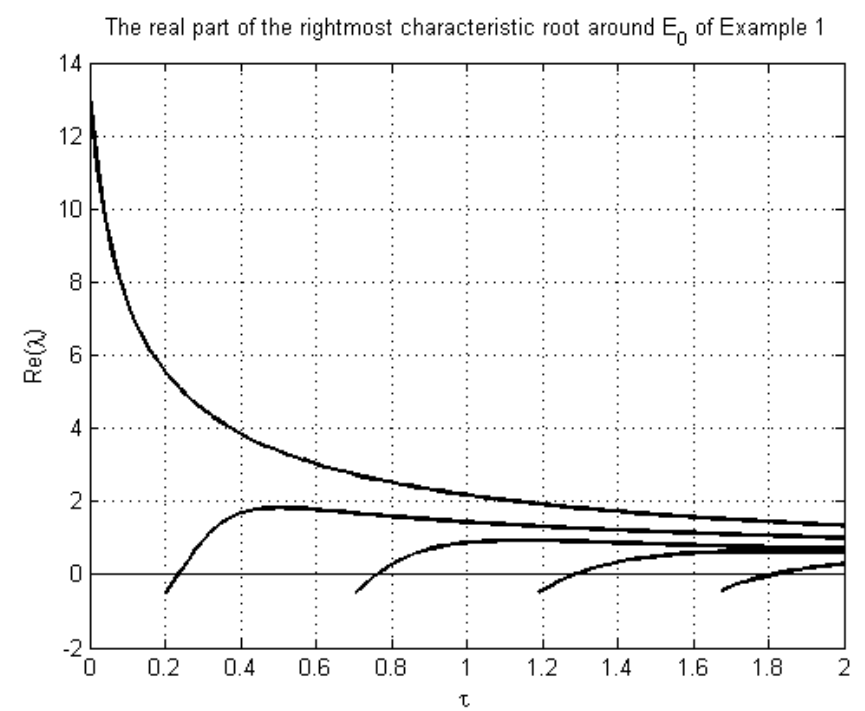

Figure 1. The rightmost root loci versus time delay.

There exist two PIRs with $\omega_{1} j=j 13.9451$ (the unstable CF) and $\omega_{2} j=j 13.0070$ (the stable CF). Accordingly, two series of critical delays are

$$
\begin{gathered}
T_{1}=\left\{\tau_{1 k} \mid \tau_{1 k}=0.31787+\frac{2 k \pi}{13.9451}\right\} \text { and } \\
T_{2}=\left\{\tau_{2 k} \mid \tau_{2 k}=0.025+\frac{2 k \pi}{13.0070}\right\}
\end{gathered}
$$

Thus, we obtain the stable switches intervals as follows:

$(0.0250,0.31787),(0.5081,0.7684),(0.9911,1.2190),(1.4742,1.6696),(1.9572,2.1201)$

$(2.4403,2.5707),(2.9234,3.0213),(3.4064,3.4718),(3.8895,3.9224),(4.3726,4.3730)$

These stable intervals are also illustrated in Figure 2. They tell us that the equilibrium point E+ has ten times the stability switches versus time delay. In addition, we draw two phase trajectories with $\tau=0.2$ (stable) and $\tau=0.4$ (unstable) in Figures 3 and 4, respectively.

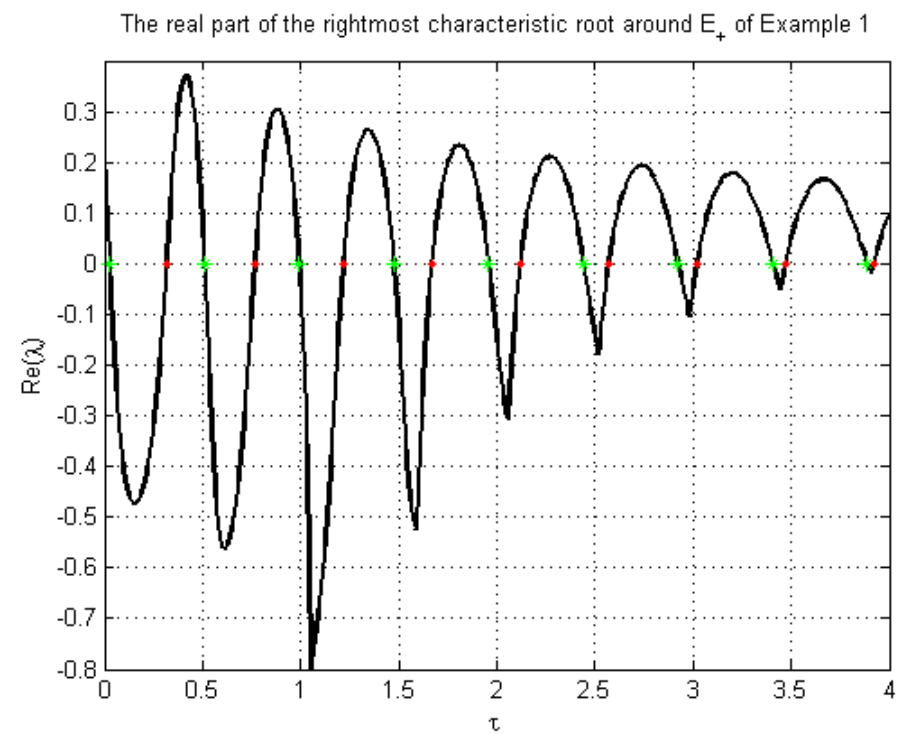

Figure 2. The rightmost root loci versus time delay. 


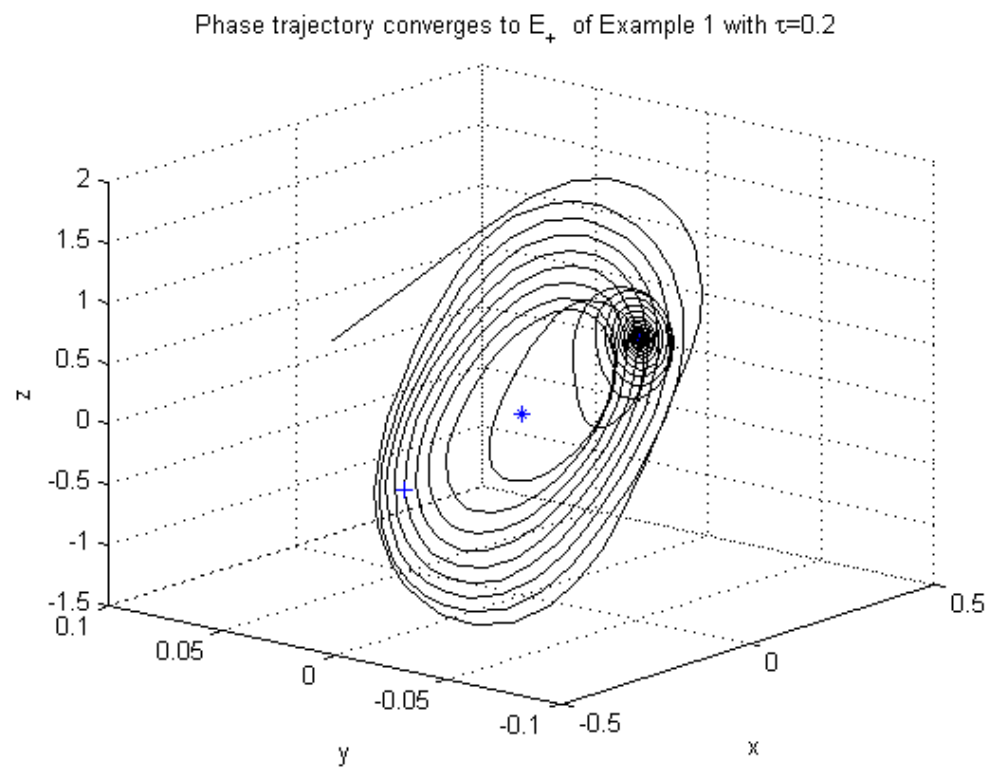

Figure 3. The stable phase trajectory.

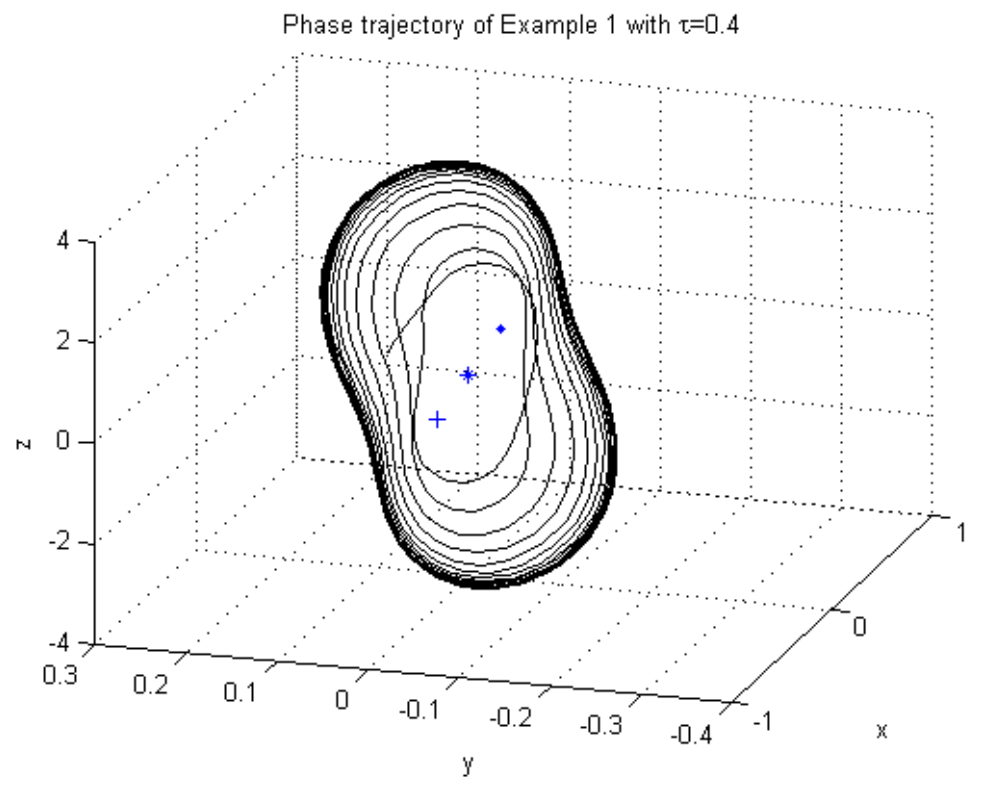

Figure 4. The unstable phase trajectory.

It should be noted that every endpoint in the intervals mentioned above is a Hopf bifurcation point, and the bifurcation analysis is determined for Example 2 in a systematic manner.

Example 2. This example is a constructed one like Example 1 with $\alpha=-0.1$. Then, there exists only one equilibrium point, $E_{0}=(0,0,0)$, and the associating characteristic equation is

$$
C E(\lambda)=\lambda^{3}+23 \lambda^{2}+160 \lambda+4000-\left(10 \lambda^{2}+30 \lambda+2000\right) e^{-\tau \lambda}
$$

Lemma 1 tells us that the exponential polynomial has two PIRs, $\omega_{1} j=j 13.6143$ and $\omega_{2} j=j 12.1149$.

The critical delays are 


$$
\begin{gathered}
T_{1}=\left\{\tau_{1 k} \mid \tau_{1 k}=0.255254+\frac{2 k \pi}{13.6143}\right\} \text { and } \\
T_{2}=\left\{\tau_{2 k} \mid \tau_{2 k}=0.0287+\frac{2 k \pi}{12.1149}\right\}
\end{gathered}
$$

Thus, we obtain the stable intervals,

$(0.0287,0.255254),(0.5473,0.7168),(1.0660,1.1783),(1.5846,1.6398)$.

which are also shown in Figure 5. Moreover, we give the stable phase trajectory with $\tau=0.1$ in Figure 6, and the bifurcation phase trajectories in Figures 7 and 8, respectively.

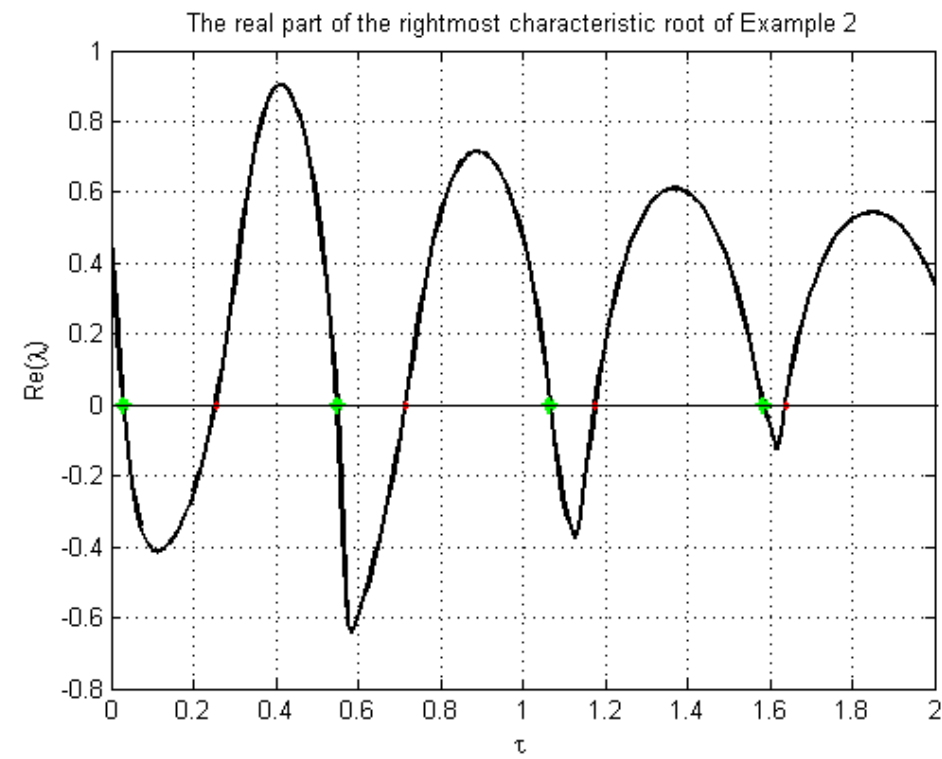

Figure 5. The rightmost root loci versus time delay.

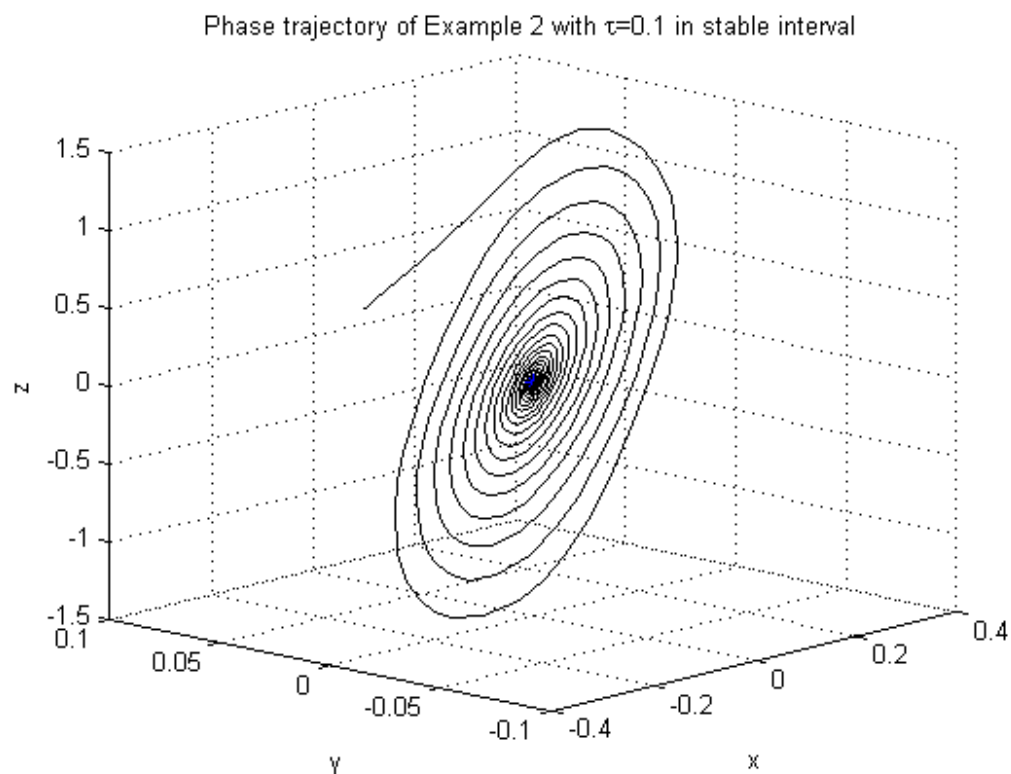

Figure 6. The stable phase trajectory. 


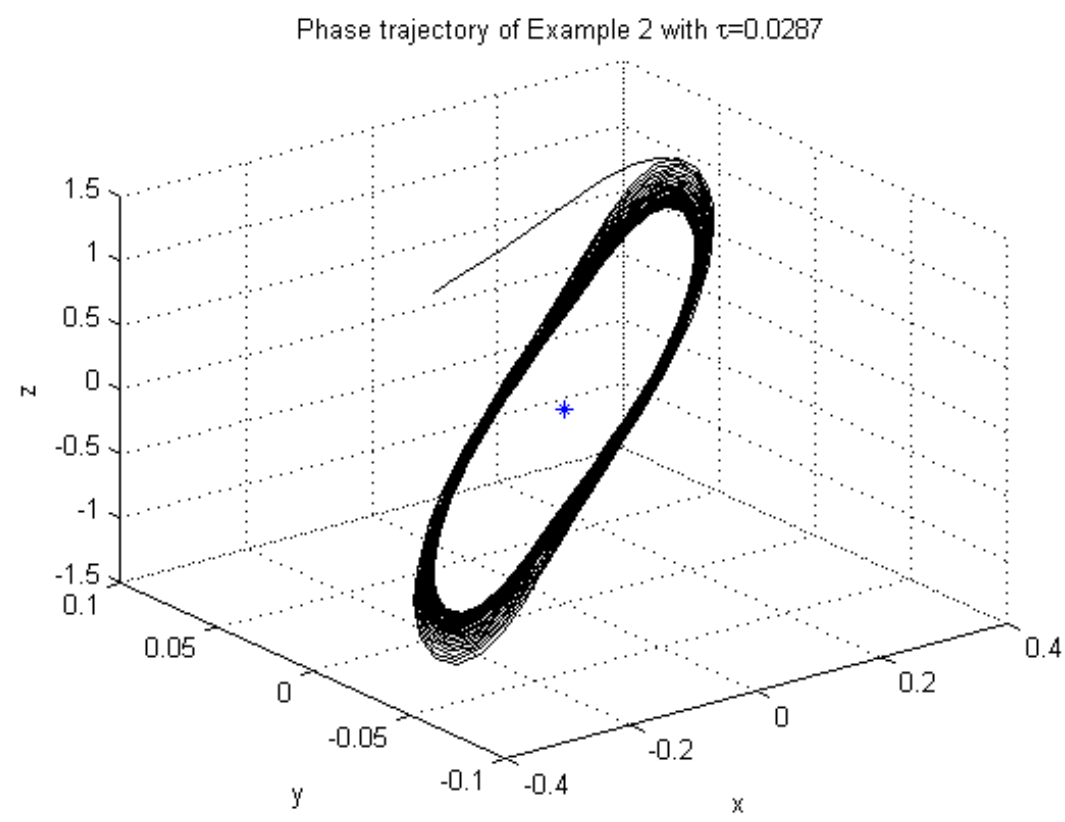

Figure 7. The bifurcation phase trajectory.

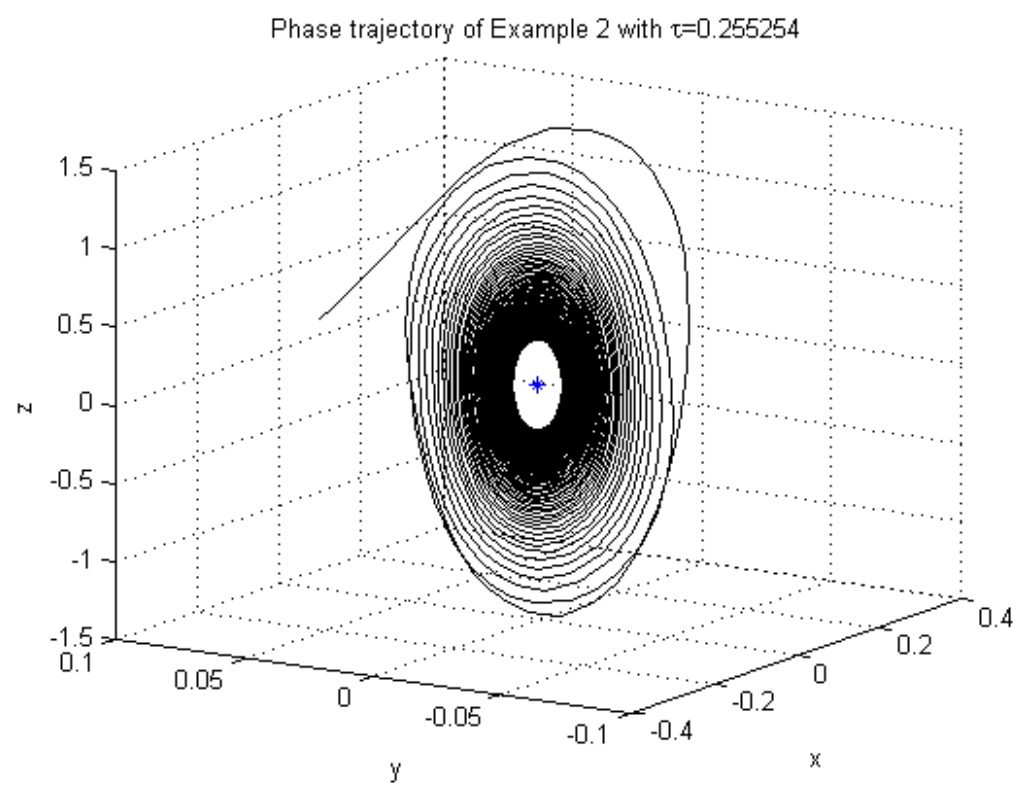

Figure 8. The bifurcation phase trajectory.

Subsequently, we choose $\tau_{10}=0.255254$ and $\tau_{20}=0.0287$ as the Hopf bifurcation points. At this time, the associating cross frequency $(C F)$ is $\omega_{1}=13.6143$ and $\omega_{2}=12.1149$, respectively. Associating with Lemma 4, Remark 3 and Theorem 2, for $\tau_{10}$, we obtain

$\bar{D}=0.2256+0.2240 j, g_{21}=-34.5439-34.3138 j, C_{1}(0)=-17.2720-17.1569 j, \alpha^{\prime}=6.04618, \omega^{\prime}=3.2116$

Thus, we have

$$
\begin{aligned}
& \mu_{2}=2.8567 \\
& \beta_{2}=-345439 \\
& \tau_{2}=0.5863
\end{aligned}
$$

Moreover, for $\tau_{20}$, we obtain 
and

$$
\begin{aligned}
& \mu_{2}=-0.5251 \\
& \beta_{2}=-13.5601 \\
& \tau_{2}=0.3064
\end{aligned}
$$

\section{Discussion}

The Lorenz system, Chua's circuit, Rossler system and the Van der Pol-Duffing equation are typical nonlinear systems. All of them can characterize the bifurcation and chaos behaviors. Indeed, they are intrinsic mathematical three-dimensional differential equations, or three-dimensional delay differential equations if there exists time delay in the states. Comparatively speaking, the nonlinear dynamics of delay differential equations are more rich than the ordinary ones. The methodology proposed in this work can be applied to other types of nonlinear circuit stems with minor changes, and more specific properties should be revealed.

\section{Conclusions}

In the paper, we have proposed a unified framework for dynamical analysis of the modified autonomous Van der Pol-Duffing equations. On one hand, the design of nonlinear circuits still needs much effort. For example, the different nonlinear parts lead to differential state response. On the other, our methodology can be improved to tackle some new nonlinear circuits. Therefore, we will pay much attention to the analysis of nonlinear dynamics among applicable circuits.

Author Contributions: T.-Y.C., methodology; H.-L.J. and H.Y., software and validation; X.-P.X., formal analysis. All authors have read and agreed to the published version of the manuscript.

Funding: This research was funded by the National Natural Science Foundation of China (NSFC), grant number 61603121.

Institutional Review Board Statement: Not applicable.

Informed Consent Statement: Not applicable.

Data Availability Statement: Not applicable.

Acknowledgments: The authors would like to thank the anonymous reviewers and their constructive suggestions for improving our work.

Conflicts of Interest: The authors declare no conflict of interest. The funders had no role in the design of the study; in the collection, analyses or interpretation of data; in the writing of the manuscript; or in the decision to publish the results.

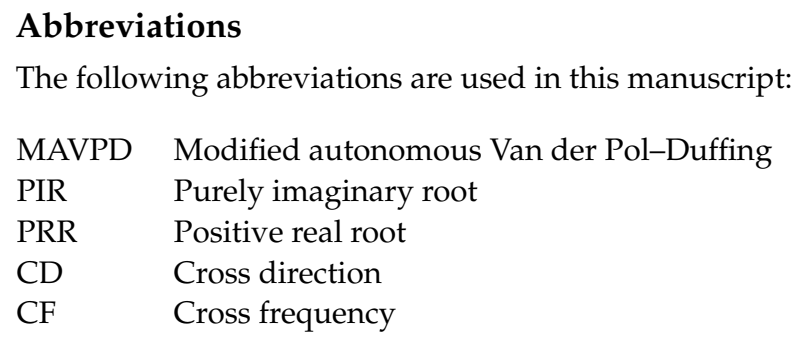

\section{Appendix A. The Proof of Lemma 2}

For intuitiveness, a diagram is introduced to illustrate the distribution of the positive real roots of cubic equation.

According to the notation in (16), $\Delta$ is the discriminant of (10). The equation has one real root with $\Delta>0$ and three distinct real roots with $\Delta<0$. Meanwhile, the origin of the coordinate system is above the cubic curve for $r<0$ and is below for $r>0$. Moreover, the $\Delta_{1}$ and $\Delta_{2}$ are the extreme points of (10). Therefore, the regions of black font, blue and red correspond to one distinct PRR, two distinct PRRs and three distinct PRRs, respectively. 


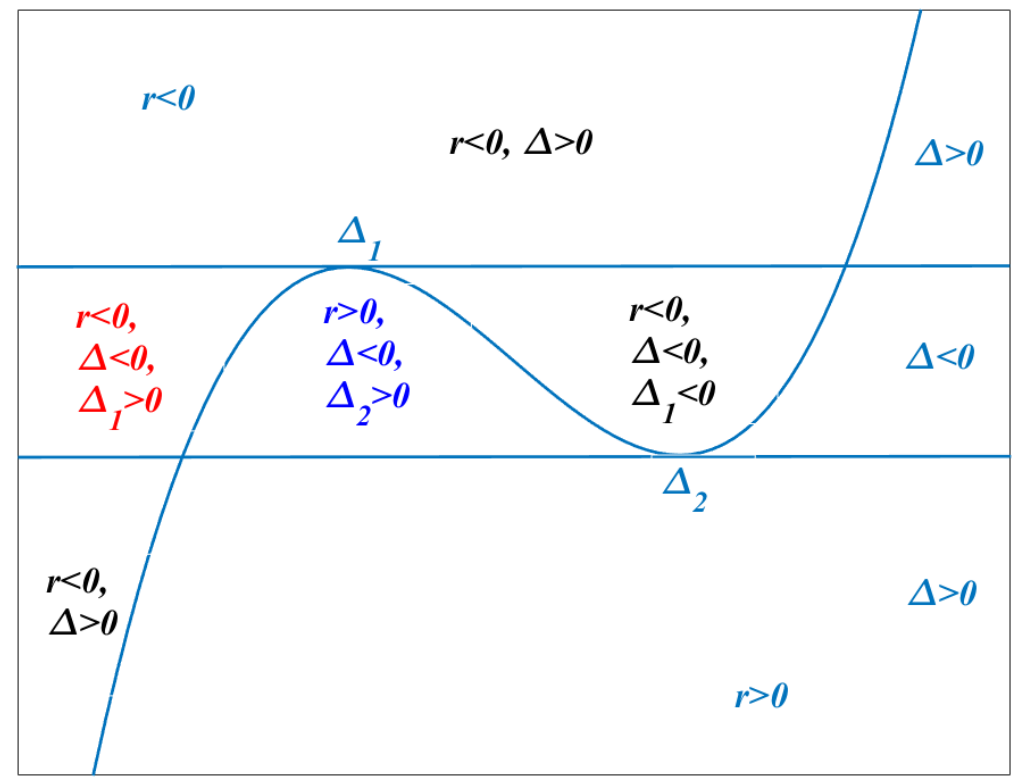

Figure A1. The distribution of PRRs of cubic equation.

On the other hand, Cardanos' formula can give three roots of (10) commonly. However, whether every root is real or complex is not determined. The types of PRRs rely on the relations of $\lambda_{1}, \lambda_{2}$ and $\lambda_{3}$ in (12) and (14). With the notations of $S$ and $T$ in (16), we have

$$
\left(\lambda_{2}+\frac{p}{3}\right)\left(\lambda_{3}+\frac{p}{3}\right)=S^{\frac{2}{3}}+T^{\frac{2}{3}}-(S T)^{\frac{1}{3}} \geq 0 .
$$

Therefore, $\lambda_{2}$ and $\lambda_{3}$ have the same sign. Either they are positive (for two distinct PRRs) in (ii) or negative (for one distinct PRRs) in (i), and case (iii) is true itself for three distinct PRRs.

\section{References}

1. Lebovitz, N.R. Bifurcation and stability problems in astrophysics. In Applications of Bifurcation Theory; Acadamic Press: New York, NY, USA, 1877; pp. 259-284.

2. Shinriki, M.; Yamamoto, M.; Mori, S. Multimode oscillations in a modified van der Pol oscillator containing a positive nonlinear conductance. Proc. IEEE 1981, 69, 394-395. [CrossRef]

3. King, G.P.; Gaito, S.T. Bistable chaos. I. Unfolding the cusp. Phys. Rev. A 1992, 46, 3092-3099. [CrossRef] [PubMed]

4. Matouk A.E.; Agiza, H.N. Bifurcations, chaos and synchronization in ADVP circuit with parallel resistor. J. Math. Anal. Appl. 2008, 341, 259-269. [CrossRef]

5. Braga, D.C.; Mello, L.F.; Messias, M. Bifurcation analysis of a Van der Pol-Duffing circuit with parallel resistor. Math. Probl. Eng. 2009, 2009, 149563. [CrossRef]

6. Fan, Q.J. Horseshoe in a modified Van der Pol-Duffing circuit. Appl. Math. Comput. 2009, 210, 436-440. [CrossRef]

7. Vincent, U.E.; Nana Nbendjo, B.R.; Ajayi, A.A.; Njah, A.N.; McClintock, P.V.E. Hyperchaos and bifurcations in a driven Van der Pol-Duffing oscillator circuit. Int. J. Dyn. Control 2015, 3, 363-370. [CrossRef]

8. Han, X.J.; Xia, F.B.; Ji, P.; Bi, Q.S.; Kurths, J. Hopf-bifurcation-delay-induced bursting patterns in a modified circuit system. Commun. Nonlinear Sci. Numer. Simul. 2016, 36, 517-527. [CrossRef]

9. Miwadinou, C.H.; Hinvi, L.A.; Monwanou, A.V.; Chabi Orou, J. Nonlinear dynamics of a $\phi^{6}$ modified Duffing oscillator: Resonant oscillations and transition to chaos. Nonlinear Dyn. 2017, 88, 97-113. [CrossRef]

10. Kumar, P.; Kumar, A.; Erlicher, S. A modified hybrid Van der Pol-Duffing-Rayleigh oscillator for modelling the lateral walking force on a rigid floor. Phys. D Nonlinear Phenom. 2017, 358, 1-14. [CrossRef]

11. Rajagopal, K.; Pone, J.; Kingni, S.T.; Arun, S.; Karthikeyan, A. Analysis and electronic implementation of an absolute memristor autonomous Van der Pol-Duffing circuit. Eur. Phys. J. Spec. Top. 2019, 228, 2287-2299. [CrossRef]

12. Rajagopal, K.; Khalaf, A.; Wei, Z.; Pham, V.T.; Alsaedi, A.; Hayat, T. Hyperchaos and coexisting attractors in a modified van der Pol-Duffing oscillator. Int. J. Bifurc. Chaos 2019, 29, 1950067. [CrossRef]

13. Ma, X.D.; Yu, Y.; Wang, L.F. Complex mixed-mode vibration types triggered by the pitchfork bifurcation delay in a driven van der Pol-Duffing oscillator. Appl. Math. Comput. 2021, 411, 126522. [CrossRef]

14. Panigoro, H.; Suryanto, A.; Kusumawinahyu, W.; Darti, I. Dynamics of an eco-epidemic predator-prey model involving fractional derivatives with power-law and mittag-leffler kernel. Symmetry 2021, 13, 785. [CrossRef] 
15. Isidori, A. Control of nonlinear systems via dynamic state feedback. In Algebraic and Geometric Methods in Nonlinear Control Theory; Riedel: Dordrecht, The Netherlands, 1986.

16. Namajunas, A.; Pyragas, K.; Tamasevicius, A. An electronic analog of the Mackey-Glass system. Phys. Lett. A 1995, 201, 42-46. [CrossRef]

17. Lee, M.S.; Hsu, C.S. On the $\tau$ decomposition method of stability analysis for retarded dynamical systems. SIAM J. Control 1969, 7 , 249-259. [CrossRef]

18. Cooke, K.; Driessche, P. On zeros of some transcendental equations. Funkcialaj Ekvacioj 1986, 29, 77-90.

19. Hassard, B.D.; Kazarinoff, N.D.; Wan, Y.H. Theory and Applications of Hopf Bifurcation; Cambridge University Press: Cambridge, UK, 1981. 